\title{
Investigation on hydrodynamic lubrication of bearings in a left ventricular assisted device
}

\author{
Xiaobing ZHENG ${ }^{1}$, Yongjian LI $^{2, *}$, Haosheng CHEN ${ }^{2}$, Shengshou HU ${ }^{1}$, Jianye ZHOU ${ }^{1, *}$ \\ ${ }^{1}$ State Key Laboratory of Cardiovascular Disease, Fuwai Hospital, National Center for Cardiovascular Diseases, Chinese Academy of \\ Medical Sciences and Peking Union Medical College, Beijing 100037, China \\ ${ }^{2}$ State Key Laboratory of Tribology, Tsinghua University, Beijing 100084, China \\ Received: 22 October 2018 / Revised: 20 March 2019 / Accepted: 08 May 2019 \\ (C) The author(s) 2019.
}

\begin{abstract}
Formations of clots were found inside the hydrodynamic bearings of the left ventricular assisted devices (LVADs) and caused tremendous risks to the long-term use of these devices. For the hydrodynamic bearings used in the LVAD, not only the lubrication status but also the motion of the blood cells in the bearing will take great effect on the performance of the device. Based on the analysis of the hydrodynamic pressures distribution and the flowing trajectory of red blood cells in the lubrication film, the bearing is designed in a region where enough hydrodynamic pressure is generated to float the rotor to reduce the wear, and the entrainment of red blood cells in the gap of the bearing can be prevented to avoid the formation of clots.
\end{abstract}

Keywords: hydrodynamic lubrication; journal bearing; left ventricular assisted device; clot

\section{Introduction}

Heart transplantation is the most successful treatment option for patients with heart failure [1], but it is now limited by a severe shortage of donor hearts. As a consequence, left ventricular assisted device (LVAD) therapy has become a treatment to provide mechanical circulatory support for the patients. Pulsatile devices (i.e., HeartMate IP1000, Thoratec PVAD, or Novacor) [2-6] are designed that cyclic filling and emptying are realized with pump actuation [7]. Newer designs of LVAD adopt rotary-pump technology to realize a continuous-flow with reduced pulsatility $[8,9]$. The continuous-flow LVAD are smaller, quieter, and more durable than pulsatile-flow devices, making them potentially better suited for long-term support [10, 11].

The formation of clots (i.e. thrombosis) is a known major and potentially fatal complication of durable continuous-flow LVAD, which may lead to requirement of subsequent pump-exchange surgery or cause premature death [12]. Considerable research effort had been made, and it had been found that clots could form and accumulate on most of the parts of LVAD such as the hub, the shroud, and the blades of the guide vane [13]. However, there is few study on the thrombosis in the LVAD bearings.

The rotor of the continuous-flow LVAD is supported by the bearings. Hydrodynamic bearing is one of the bearings most commonly used in LVAD. In the design of this kind of bearings, sufficient hydrodynamic pressure needs to be generated in the lubrication film to separate the pin of the rotor from the bearing to reduce the friction and the wear. For the journal bearing, the load mainly comes from the weight of the rotor, which is relatively small for the LVADs, and the bearings should work under the full-film lubrication condition in most cases. However, in the LVADs, blood cells, such as red blood cells (RBCs), might flow inside and accumulate in the gap of the bearing to form clots. Once the clots have formed and accumulated in the gap of the bearings, the lubrication state of the bearing will be changed. This may lead to severe

* Corresponding authors: Jianye ZHOU, E-mail: zhoujianye@fuwaihospital.org; Yongjian LI, E-mail: liyongjian@tsinghua.edu.cn 
wear between the pin of rotor and the bearing, or even cause an unexpected stop of the rotor during its working time. Therefore, the design of the bearing is still a challenge and on an urgent demand in the rapid development of LVADs.

In this work, a LVAD (Fuwai Hospital, Chinese Academy of Medical Sciences) is taken for example for the design of the bearing. Under the given size of the bearing and the rotation speed of the rotor, an optimized region for the rotor's pin size is provided based on the analysis of hydrodynamic pressure and the motion of RBCs. Within the region, a full film lubrication is achieved with enough hydrodynamic pressure to float the rotor, and the entrainment of the RBCs is avoided to prevent the formation of clots.

\section{Experiment and numerical simulation}

\subsection{Experiment}

The LVAD pump is implanted in the test sheep. The implanted pumps draw blood from the apex of the left ventricle and deliver it to the ascending aorta, as shown in Fig. 1(a). The left ventricular assist device is capable of a flow rate up to 5 liters per minute at a mean pressure of $128 \mathrm{~mm} \mathrm{Hg}$.

The device consists of four main parts, which are the front guide vane, the impeller rotor, the rear guide vane, and the outer stator with coils and cores, as shown in Fig. 1(b). The 3D structure of the impeller rotor and the guide vanes are illustrated in Fig. 1(c).

The weight of the impeller rotor is $16 \mathrm{~g}$. It is supported by two bearings, the structure of which is shown in Fig. 1(d). The bearings are installed in the front and the rear guide vane hubs, respectively. Both of the two bearings have trust part and journal part made of artificial gemstone. The steel pins on each end of the impeller rotor are inserted into the bearing to support the rotor. The permanent magnets are installed in the impeller rotor. The electric currents in the stator coils change regularly under the control of a transistor and the magnetic field vary accordingly, which drive the permanent magnets, along with the impeller rotor, to rotate. The blades on the rotor will drive the blood in the pump shell to assist the heart to pump the blood.

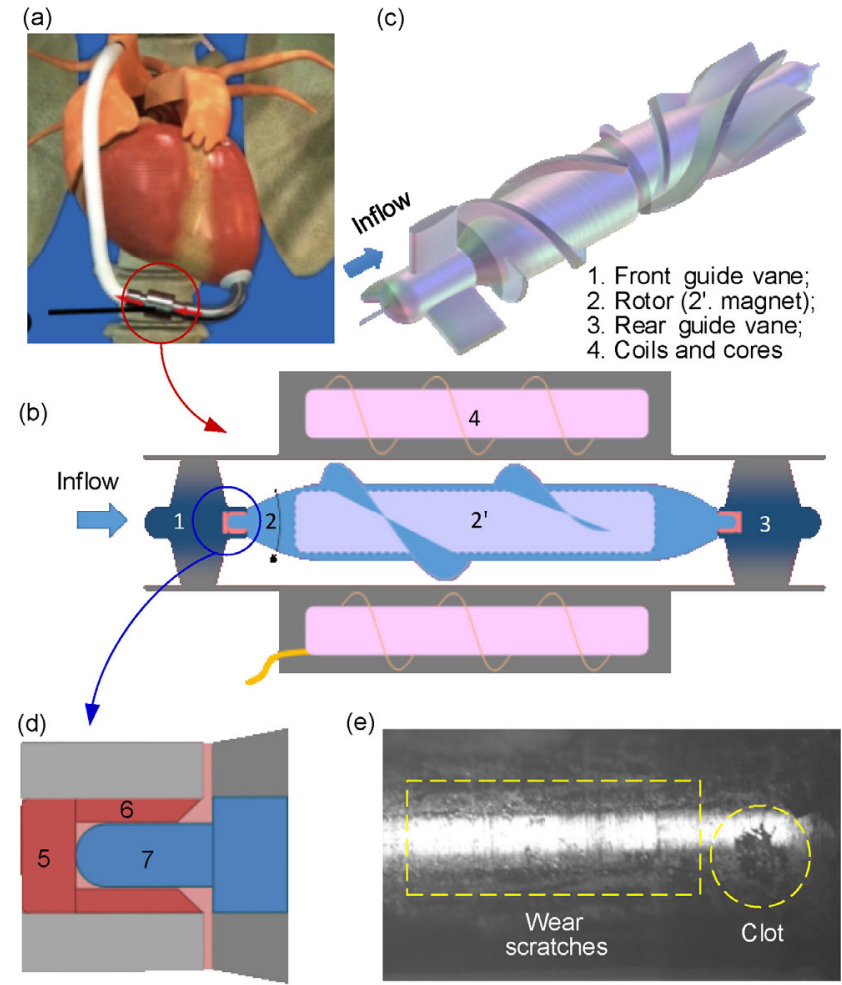

5. Thrust bearing; 6. Journal bearing; 7. Pin

Fig. 1 The structures of the LVAD and the bearing. (a) Schematics of the LVAD in the body (sheep), (b) main structure of the LVAD, (c) 3D structure of the impeller rotor and guide vanes, (d) the bearing structure, (e) view of the pin surface (with wear scratches and clot marks) after the test in the body.

The pumps were implanted in the sheep, and worked for about one week before they were taken out. Then, the pins of the pumps were observed and measured with the optical microscope, 3 dimensional confocal microscope (Phase Shift MicroXAM-3D) and scanning electron microscope (FEI Quanta 200 FEG). Figure 1(e) shows that wear scratches and clot marks were found on surface of the pin after the test in the sheep.

\subsection{Numerical simulation method}

To design the bearing, the hydrodynamic pressure in the lubrication film is to be calculated. Here, a 3D model of the bearing with finite width is established, as shown in Fig. 2(a). The fluid in the gap of the bearing is taken into account, including both of the radial clearance and axial clearance between the bearing and the pin. A numerical method is adopted to calculate the pressure distribution of the lubrication film. The grid generation is performed using the mesh module of the ANSYS (ANSYS, Inc.) The grids are structure- 

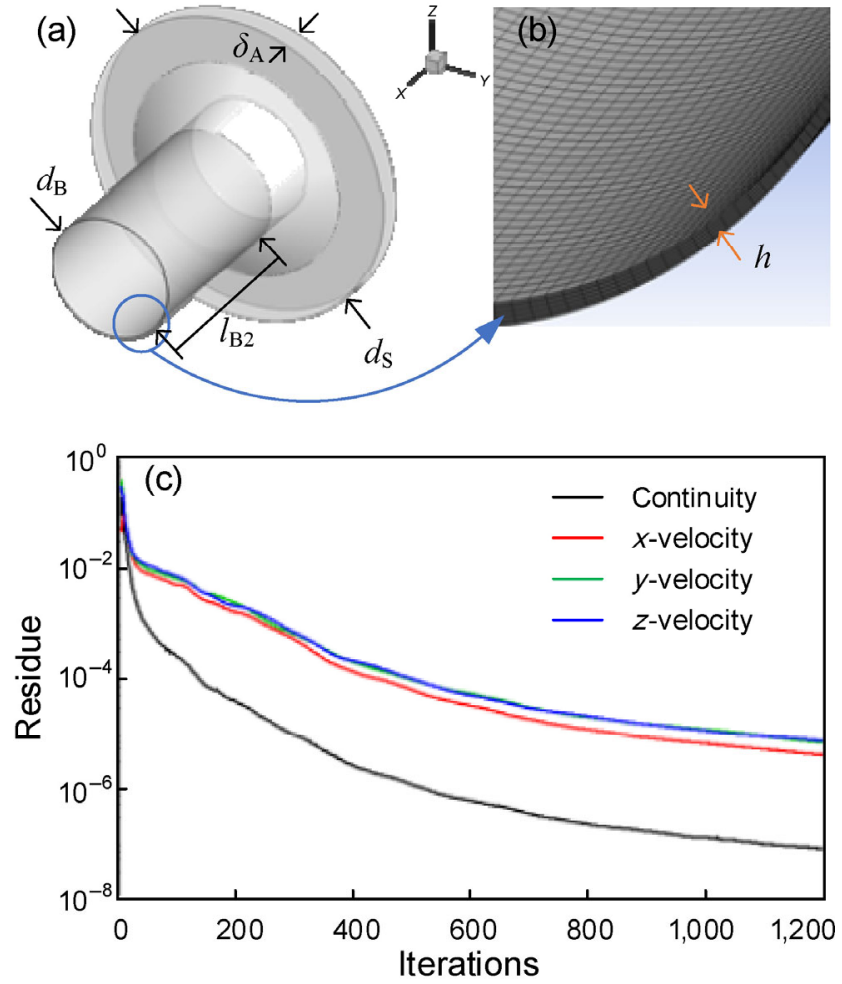

Fig. 2 Geometry model for the numerical calculation of the pressure distribution on the pin. (a) The geometry model of the pin, (b) the grid on the thin lubrication film, (c) the residue of the calculation during the iterations of the numerical method.

grid type with the minimal grid size of $0.2 \mu \mathrm{m}$ in the thickness direction, as shown in Fig. 2(b). The thickness of the lubrication film $h$ is determined by the inner diameter of the journal bearing $d_{\mathrm{B}}$ and the diameter of the pin $d_{\mathrm{p}}$. Here, $d_{\mathrm{B}}$ is a given parameter, and the parameter of $d_{\mathrm{p}}$ is to be designed to adjust the hydrodynamic pressures in the numerical calculation.

The calculations are performed based commercial software ANSYS-Fluent (ANSYS, Inc.). The viscosity of the blood $\eta=1.0 \mathrm{mPa} \cdot \mathrm{s}$ and the density $\rho=1.0 \mathrm{~g} / \mathrm{cm}^{3}$. The other working conditions such as the rotating speed $\omega$, the size of the bearing $d_{\mathrm{B}}$, etc., are presented in Table 1. The Reynolds number of the flow under the given conditions is $4-20$ so the calculations are conducted in a laminar flow condition. The governing equations for the calculation of the pressures are mass and momentum conservation equations, as presented in Eqs. (1) and (2), where $x_{1}, x_{2}, x_{3}$ are the $x, y, z$ in Cartesian coordinates, respectively; $u_{1}, u_{2}, u_{3}$ are the velocities in $x, y, z$ directions, respectively; $\rho$ is the density of the fluid; $\eta$ is the viscosity of the fluid; and $p$ is the pressure.

$$
\begin{gathered}
\sum_{i=1}^{3} \frac{\partial\left(\rho u_{i}\right)}{\partial x_{i}}=0 \\
\sum_{j=1}^{3} \frac{\partial\left(\rho u_{i} u_{j}\right)}{\partial x_{j}}=\eta \sum_{j=1}^{3} \frac{\partial^{2} u_{i}}{\partial x_{j}^{2}}-\frac{\partial p}{\partial x_{i}}
\end{gathered}
$$

During the application of LVADs, the exact operation condition varies as the change of posture, motion, and the physiology of the body. In this study, we focus on the basic design of the bearings only taking the hydrodynamic effect and the motion of the RBCs into account, so we had simplified the situation to a series of steady cases with different given lubrication film thicknesses and the rotor was kept horizontal.

The boundary conditions for the calculation are as following: the inner surface of the lubrication film is a moving wall with the speed of $\omega$, the outer surface of the film is a static wall. The opening end of the film connected to the outer flow is treated as a pressure inlet, while the end to the thrust bearing is treated as a static wall. In the calculation of the pressure distribution, we apply the Reynolds boundary condition, for which the negative pressure was set zero to represent the rupture of the lubricant film when the pressure reach zero. In fact, the rupture of the lubricant film should depend on the absolute pressure in the film, which is affected by the pressure in surrounding region of the bearings. In the LVADs, the bearing at the front guide vane should be a bit lower than the atmospheric pressure, while that at the rear guide vane is about $128 \mathrm{~mm} \mathrm{Hg}(16 \mathrm{kPa})$ higher for the LVAD in this study. We had assumed a surrounding pressure of one atmospheric pressure (about $100 \mathrm{kPa}$ ) in the simulations.

Table 1 Parameters and values for the calculation.

\begin{tabular}{ccc}
\hline Name & Explanation & Value \\
\hline$\omega$ & Rotating speed of pin & $10,000 \mathrm{rpm}$ \\
$d_{\mathrm{B}}$ & Diameter of bearing & $1.61 \mathrm{~mm}$ \\
$d_{\mathrm{p}}$ & Diameter of pin & $1.56-1.60 \mathrm{~mm}$ \\
$d_{\mathrm{S}}$ & Diameter of leading surface of rotor & $2.80 \mathrm{~mm}$ \\
$l_{\mathrm{B} 2}$ & Length of bearing & $2.30 \mathrm{~mm}$ \\
$\delta_{\mathrm{A}}$ & Axial clearance between bearing & $0.3 \mathrm{~mm}$ \\
& and rotor \\
$R$ & Radius of the magnetic rotor \\
$L$ & Length of the magnetic rotor & $4.25 \mathrm{~mm}$ \\
$\delta_{0}$ & Average "air" gap & $20 \mathrm{~mm}$ \\
$\mu_{0}$ & Magnetic permeability coefficients & $4 \pi \times 10^{-7} \mathrm{H} / \mathrm{m}$ \\
$F_{\mathrm{j}}$ & Fundamental magnetic motive force & $130.06 \mathrm{~A}$ \\
\hline
\end{tabular}


Finite-volume method (FVM) is applied to discretize the governing equations. Semi-Imaplicit Method for Pressure Linked Equations-Consistent (SIMPLEC) scheme is utilized for pressure-velocity coupling. For spatial discretization, PREssure STaggering Option (PRESTO!) scheme and first order upwind scheme are used for the pressure equations and momentum equations, respectively [14]. The convergence criterion is set as $10^{-5}$, so that the results are precise and stable. In this work, the residue during the calculation process is presented in Fig. 2(c).

For the journal bearing, the load mainly comes from the weight of the rotor and the unbalanced magnetic force. For steady study, the unbalanced magnetic forces due to the rotor static eccentricity were calculated according to Eq. (3), where, $R$ and $L$ are the radius and the length of the magnetic rotor, respectively, $\delta_{0}$ is the average thickness of the gap, $F_{\mathrm{j}}$ is the fundamental magnetic motive force, $\mu_{0}$ is the magnetic permeability coefficients, and it can be expressed as the Fourier series expansion with the Fourier coefficients, $\Lambda_{i}$, which are presented in Eq. (4). And $\varepsilon$ is the eccentricity rate of the rotor relative to the outer stator.

$$
\begin{gathered}
F=\frac{R L \pi}{4 \mu_{0}} F_{j}^{2}\left(\Lambda_{0} \Lambda_{1}+\Lambda_{1} \Lambda_{2}+\Lambda_{2} \Lambda_{3}\right) \\
\begin{cases}\Lambda_{i}=\frac{\mu_{0}}{\delta_{0}} \frac{1}{\sqrt{1-\varepsilon^{2}}} & (i=0) \\
\Lambda_{i}=\frac{2 \mu_{0}}{\delta_{0}} \frac{1}{\sqrt{1-\varepsilon^{2}}}\left(\frac{1-\sqrt{1-\varepsilon^{2}}}{\varepsilon}\right)^{i} & (i>0)\end{cases}
\end{gathered}
$$

Within the scope of this test, the unbalanced magnetic force due to the rotor static eccentricity is less than $1 \%$ of the load that is caused by the weight of the rotor. So the unbalanced magnetic force is neglected in the subsequent analysis.

The motion of the RBCs are analyzed with the discrete phase model (DPM) [15]. The RBCs are treated as spherical particles. The force equilibrium equation of particles in Cartesian coordinates is presented in Eq. (5) [15], where, $u_{\mathrm{p} i}$ is the particle velocity in $x_{i}$ direction, $\eta_{\mathrm{p}}$ is the density of the particle, $F_{\mathrm{D}}, g_{x i}$ and $F_{x i}$ are the drag force, the gravity and the sum of the other forces acting on the particle in $x_{i}$ direction, respectively.

$$
\frac{\mathrm{d} u_{\mathrm{p} i}}{\mathrm{~d} t}=F_{\mathrm{D}}\left(u_{i}-u_{\mathrm{p} i}\right)+\frac{g_{x i}\left(\rho_{\mathrm{p}}-\rho\right)}{\rho_{\mathrm{p}}}+F_{x i}
$$

With the DPM method, the trajectory of the particles were obtained and the motion of RBCs in the bearing are analyzed.

According to the calculation results, two objectives on the design of the bearing are investigated. The first is the size of the pin. The increase of the pin size will decrease the lubrication film thickness, which would generate higher hydrodynamic pressure and help to balance the radial load. In this objective, the surface roughness of the pin also needs to be considered since it would reduce the actual gap between the pin and the bearing. The second objective is to investigate whether RBCs can enter and stay in the gap or not.

\section{Results and discussion}

\subsection{Pressures and surface roughness}

The distribution of the hydrodynamic pressure in the lubrication film are presented in Figs. 3(a) and 3(b) in a static and a running condition, respectively. The pressure presents an asymmetric distribution on the running pin, which indicates the hydrodynamic pressure is generated and it will balance the weight of the rotor. With the given running speed and the size of the bearing $d_{\mathrm{B}}=1.61 \mathrm{~mm}$, the hydrodynamic pressure is mainly affected by the size of the pin $d_{\mathrm{p}}$. The hydrodynamic pressure distributed on the pin surface with different minimum film thickness $h_{\min }=$ $0.5\left(d_{\mathrm{B}}-d_{\mathrm{p}}\right)-e$ is presented in Fig. 3(c). It is found that the pressure increases as the $h_{\min }$ decreases.

Integrating the hydrodynamic pressures all around the pin surface, the load capacity provided by the hydrodynamic pressure can be acquired according to Eq. (6) [16].

$$
F_{\mathrm{C}}=\frac{\omega d_{\mathrm{p}} \eta L^{3} e}{\left(d_{\mathrm{B}}-d_{\mathrm{p}}\right)} \sqrt{\frac{\left(64-4 \pi^{2}\right) e^{2}+\pi^{2}\left(d_{\mathrm{B}}-d_{\mathrm{p}}\right)^{2}}{\left[\left(d_{\mathrm{B}}-d_{\mathrm{p}}\right)^{2}-4 e^{2}\right]^{3}}}
$$

The calculated load capacity under different minimum film thickness $h_{\min }$ is illustrated in Fig. 3(d). To balance the weight of the rotor and realize the full film lubrication, the ratio of the load capacity to the 

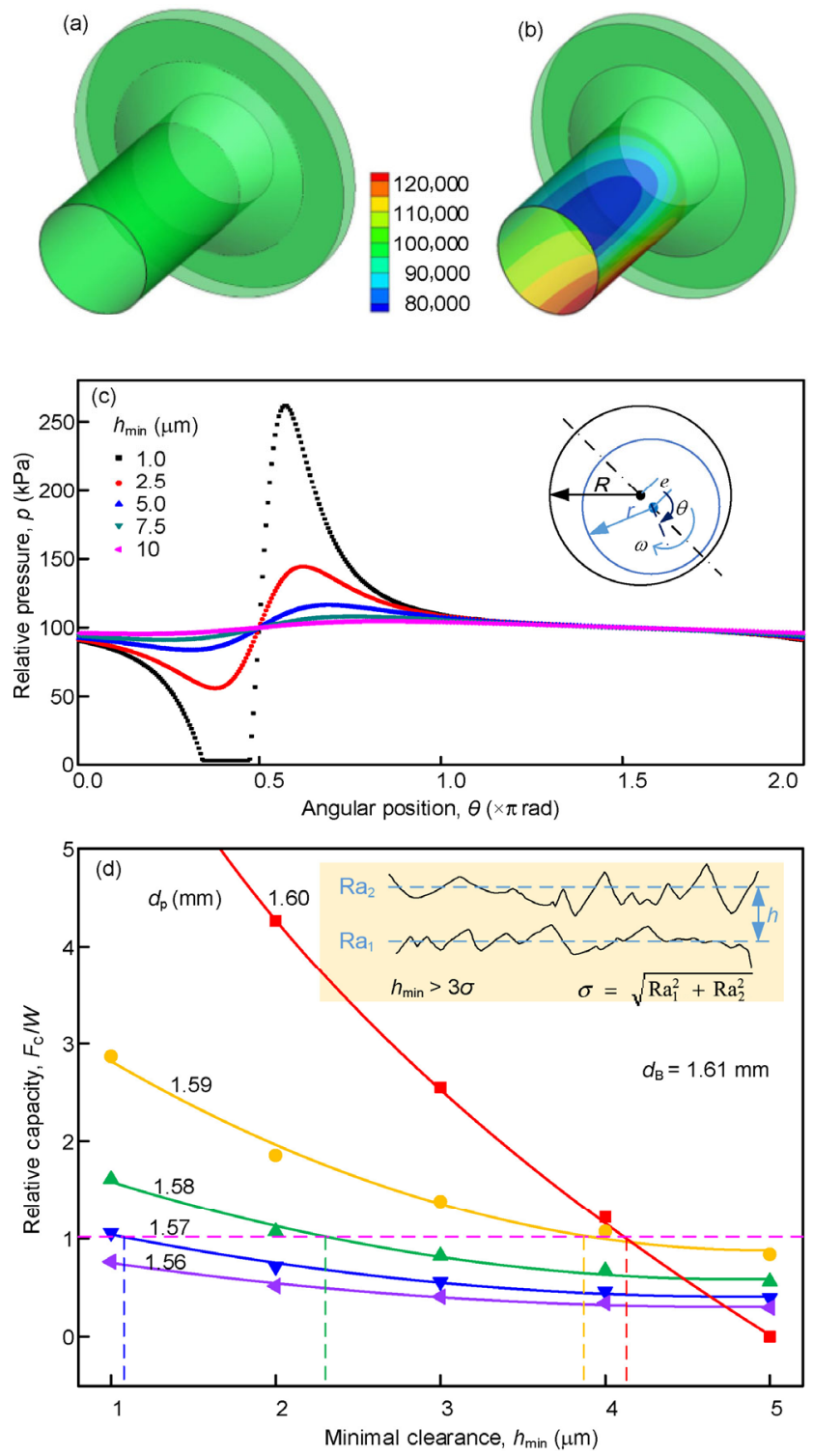

Fig. 3 Calculated hydrodynamic pressure in the bearing. (a) and (b) show a $3 \mathrm{D}$ view of the pressure distribution in the bearing in static and running conditions, respectively. (c) is the pressure distribution along the perimeter of the pin with different minimal clearance. The insert schematics illustrate the status of the pin under the lubrication. (d) The load capacity varied with the minimum clearance $h_{\min }$ under different pin size. The dash line is the critical value of the load capacity to float the rotor. The inserted schematics show the actual clearance between the rough surfaces.

weight of the rotor $F_{d} / W$ should be larger than 1 . From the calculated results, it is found that a smaller $h_{\min }$ with the same pin size provides higher load capacity, and the critical $h_{\min }$ to float the rotor is then determined when $F_{\mathrm{c}} / W=1$, as illustrated by the dash line in Fig. 3(d). In this figure, it is also found that a larger pin size $d_{\mathrm{p}}$ would cause higher load capacity under the same $h_{\min }$.
Thus, the critical $h_{\min }$ increases as the pin size increases. For example, the critical $h_{\min }$ is $2.2 \mu \mathrm{m}$ for the pin with the size $d_{\mathrm{p}}$ of $1.58 \mathrm{~mm}$, while it increases to $3.9 \mu \mathrm{m}$ when the pin size $d_{\mathrm{p}}$ is $1.59 \mathrm{~mm}$. The variation of $h_{\min }$ indicates that a larger pin is better to provide enough load capacity in the lubrication film to float the rotor, while a smaller pin needs a larger eccentricity $e$ to achieve a smaller $h_{\min }$ to get enough load capacity. When a pin is too small, the $h_{\min }$ would become too small to be realized, which is demonstrated by the curve of $d_{\mathrm{p}}=1.56 \mathrm{~mm}$.

According to the predicted critical $h_{\min }$ the size of the pin can be determined. But in practice, the minimal clearance also needs to satisfy the condition $h_{\min }>3 \sigma$ to prevent the contact of two rough surface [17], where $\sigma$ is the mean square value of the surface roughness of the two solid surfaces, $\sigma=\left(\mathrm{Ra}_{1}{ }^{2}+\mathrm{Ra}_{2}{ }^{2}\right)^{1 / 2}$, as illustrated by the inserted schematics in Fig. 3(d). Based on the calculated results, the pin size is better to be larger to acquire larger $h_{\min }$ to avoid the contact of two rough surfaces. But there is an upper limitation on the pin size since the increasing pin size would reduce the gap in the bearing. For example, when the pin size reaches $1.60 \mathrm{~mm}$, the critical $h_{\min }$ is about $4.1 \mu \mathrm{m}$, which is larger compared to that from the pin size of $1.59 \mathrm{~mm}$. But when the pin size is $1.605 \mathrm{~mm}$, the $h_{\min }$ would be less than $2.5 \mu \mathrm{m}$ and it requires a lower surface roughness compared to that for the pin size of $1.60 \mathrm{~mm}$.

On the other hand, the surface roughness of the pin can be determined for a given pin size according to the relationship $\mathrm{Ra}_{2}<\left(\left(h_{\min } / 3\right)^{2}-\mathrm{Ra}_{1}{ }^{2}\right)^{1 / 2}$. For example, when $\mathrm{Ra}_{1}=1 \mu \mathrm{m}, h_{\min }=2.2 \mu \mathrm{m}$ for $d_{\mathrm{p}}=1.58 \mathrm{~mm}, \mathrm{Ra}_{2}$ is derived as $1.8 \mu \mathrm{m}$, and the corresponding surface treatment method such as polishing and grinding would be determined.

\subsection{RBCs in the clearance}

To design the pin, another condition needs to be considered is that the RBCs will not be pulled into the gap in the bearing. In a lubricated status, the pin will not stay at the center of the bearing because of the eccentricity as shown by the inserted schematics in Fig. 3(c). Therefore, the position opposite to the minimal clearance will have the maximum gap. For example, when $d_{\mathrm{p}}=1.59 \mathrm{~mm}$, and $h_{\min }=2.2 \mu \mathrm{m}$ from the results in Fig. 3(d), the $h_{\max }=27.8 \mu \mathrm{m}$, which is much larger 
than the average diameter $8 \mu \mathrm{m}$ of RBCs. This gap would allow the deformable RBCs to flow inside. On the other hand, there are high and low pressure regions caused by the hydrodynamic pressure distribution, as presented in Fig. 3(c). The low pressure provides the force to pull RBCs into the gap. The trajectory of a RBC can be predicted by the calculated streamlines in the lubrication film, and it may have three different status with the different pin size, as presented in Figs. 4(a) -4 (c).

The first status is that the RBCs are pulled into the gap with a relative large depth along the axis. RBCs are circulating in the gap and passing the high and low pressure regions periodically, as show in Fig. 4(a). The circulation and accumulation in the gap will cause the blood clot on the pin surface. Actually, the entrainment of RBCs into the gap occurred in the experiments, and the formed clots on the surface of the pin are found, as shown in Figs. 4(c) and 4(d). The second status is that the RBCs can flow into the gap, but within a few rounds they are pushed out of the gap and they are not accumulated in the gap, as shown in Fig. 4(b). With the increase of the pin size, the third status occurs, where the RBCs are not entering the gap, and they are only circulating at the entrance of the gap, as shown in Fig. 4(c). In the latter two statuses, the RBCs would not accumulate in the gap, and there will be no clots formed on the pin surface. In the experiment, the pin surface is clean and no adhesion of the RBCs is found for the latter two conditions, as shown by Fig. 4(e).

It is found that the first status occurs when the pin size and the eccentricity are both smaller. With the increase of the pin size and the eccentricity, the flowing RBCs gradually changes to the second and the third status. The streamlines in the gap with the change of the pin size and the minimal clearance $h_{\min }$ are simulated, and the results are presented in Fig. 4(f) (to be consistent with the results from Fig. 3, here the eccentricity $e$ is presented by the $h_{\min }$ and $h_{\min }=$ $\left.0.5\left(d_{\mathrm{B}}-d_{\mathrm{p}}\right)-e\right)$. The $2^{\text {nd }}$ and $3^{\text {rd }}$ status for the RBCs flowing in the gap are within the boundaries of dashed lines, as shown in Fig. 4(f). According to the results, the proper pin size together with the eccentricity to avoid entrainment of RBSs in the gap can be determined. To prevent the entrainment of RBCs in the gap, a practical method is to increase $d_{\mathrm{p}}$ which reduces both the gap size and the eccentricity.

Here, the streamlines of the continuous fluid are used to represent the trajectory of the RBCs. Usually, the RBCs are flowing along the streamlines because they are small and have high deformability [18]. In this simulation the RBCs are considered here to follow the streamlines with their high deformability and the minimal gap is set to 3 microns, which is equal to the thickness of a RBC to allow the flowing of $R B C$ in the gap.

\subsection{Optimization of bearing design}

According to the calculated results of hydrodynamic
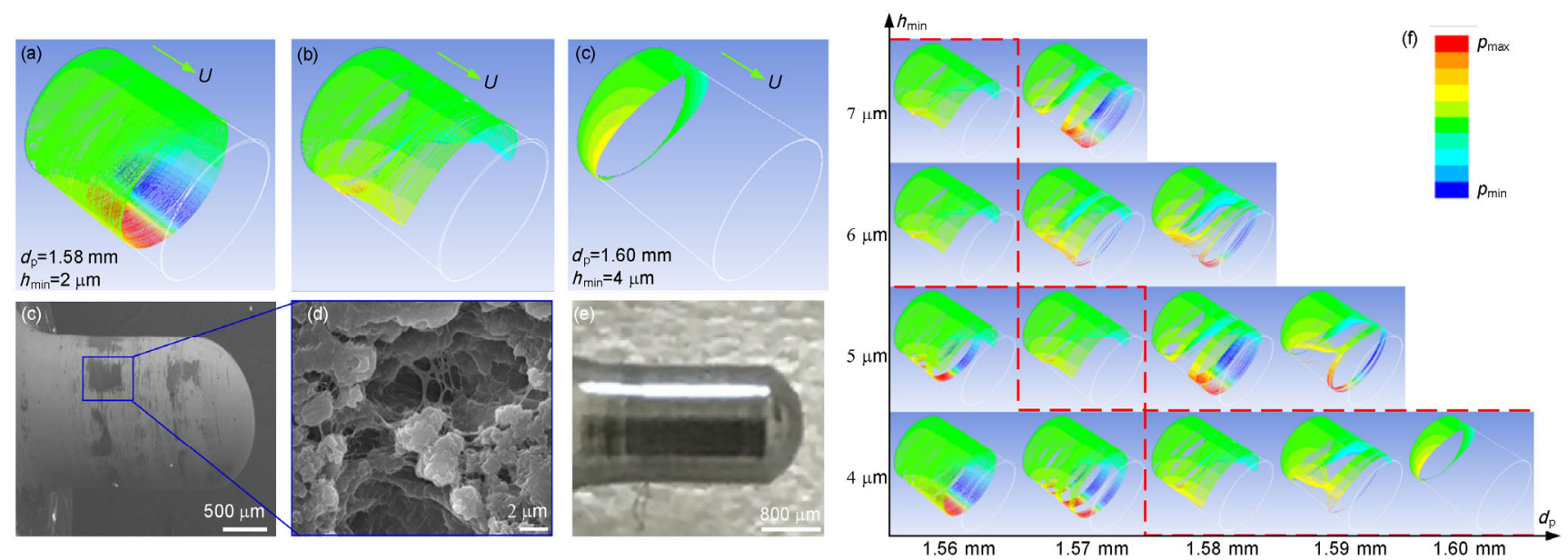

Fig. 4 Flowing status of RBCs in the gap. (a)-(c) show the calculated streamlines in the gap, corresponding to the three different status of the entrainment of RBCs, (c) and (d) show the clots formed on the pin surface in the experiment, while (e) shows the clean surface of the pin, (f) the streamlines in the gap changed with the pin size and the minimal clearance. 
pressures and the streamlines of the RBCs in the gap, the pin size and the minimal clearance (or the eccentricity of the shaft) are the crucial parameters to affect the performance of the bearing. Here we draw a diagram of the bearing performance as shown by Fig. 5 where the load capacity and the entrainment of the RBCs in the gap are dependent of the pin size and the minimal clearance. In Fig. 5, the blue lines are the contour of relative load capacity, which is from

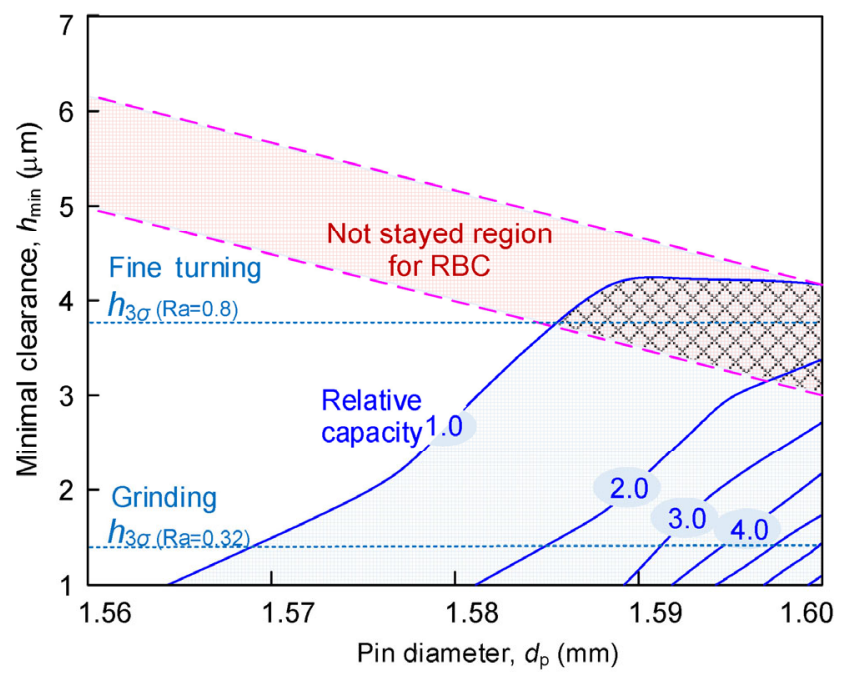

Fig. 5 The diagram of the bearing performance on the relative load capacity and the entrainment of RBCs under different pin diameter and minimal clearance. the results shown in Fig. 3(d). The red region between the two dashed lines is the no entrainment region of RBCs which is from the simulations results in Fig. 4(f). The blue dotted lines illustrate the corresponding surface treatment method on the pin, and the acquired surface roughness Ra can satisfy the minimal clearance.

The superposed region with shadows is the optimized parameters of pin size and the minimal clearance for the design of the bearing. In the region, the hydrodynamic pressure can float the rotor, while the RBCs would not circulate and accumulate in the gap to form the clots.

The design of the bearing is crucial to the performance and the working life of the device. For the original design with the pin diameter of $1.58 \mathrm{~mm}$, which was outside of the superposed region, distinct wear scratches were found on the surface of the pin after the 168 hours running test in the sheep body, as shown in Figs. 6(a) and 6(b). For the optimal design with the pin diameter of $1.60 \mathrm{~mm}$, the pin surface is still smooth after the test, as shown in Figs. 6(c) and 6(d), and no clot marks are found as shown in Fig. 4(e).

\section{Conclusions}

In this work, the hydrodynamic pressures generated
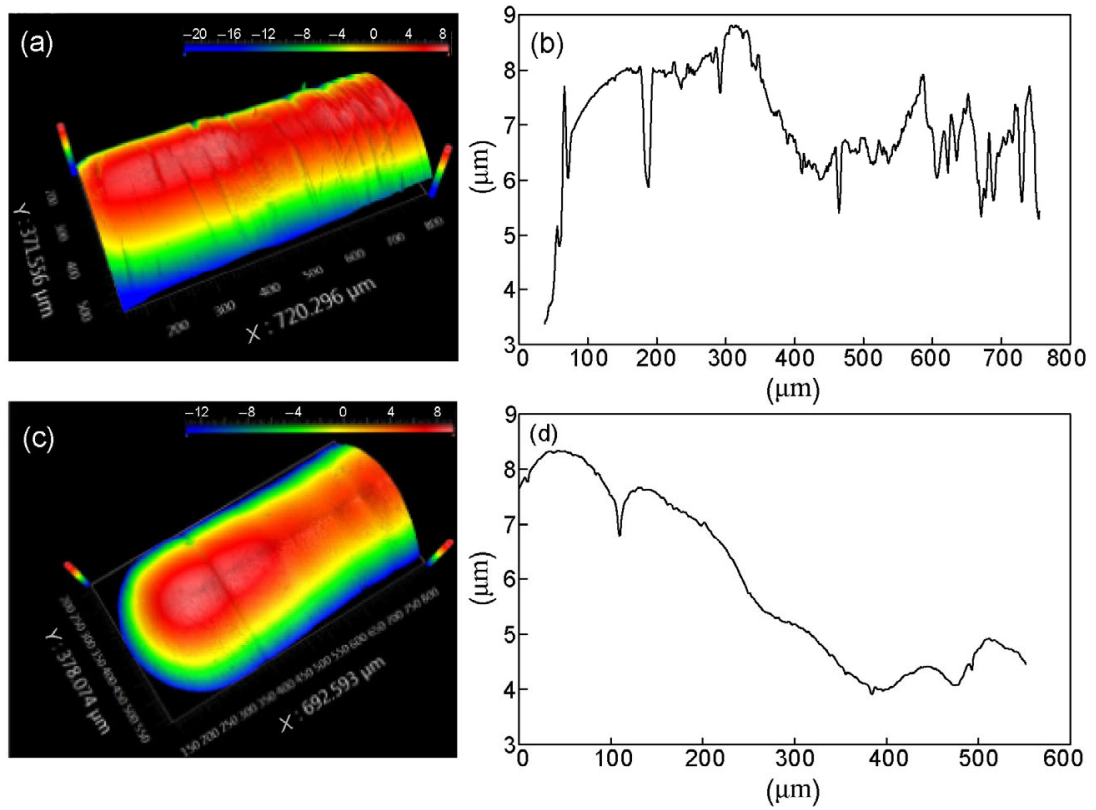

Fig. 6 Surface profile of the pin after the tests (a) and (b) are the surface profile of the pin in a tight fit in 3D and 2D view, respectively, and scratches are found on the surface after a 168-hour running experiment in the body (sheep). (c) and (d) are the surface profile of the pin in a well-designed fit in 3D and 2D view, respectively, and the surface is still smooth after the experiment. 
in the lubrication film in the bearing of a LVAD are numerically analyzed. Based on these numerical results, the size of the pin of the rotor is designed under a given bearing size and rotating speed. The pin should be large to generate enough hydrodynamic pressures to float the rotor, while it should not beyond the size that causes the contact of the two rough surfaces. Also, larger pin would help to prevent the entrainment of the RBCs in the gap of the bearing. Taking into all the three effects, a region for the design of the pin in a given bearing is provided. This demonstration may be helpful in the design of a LVAD to reach longer working life with better performance.

\section{Acknowledgements}

This work is supported by the National Key R \& D Program of China (2017YFC0111105), and the funding of State Key Laboratory of Tribology, China (SKLT2018B03).

Open Access: This article is licensed under a Creative Commons Attribution 4.0 International Li-cense, which permits use, sharing, adaptation, distribution and reproduction in any medium or for-mat, as long as you give appropriate credit to the original author(s) and the source, provide a link to the Creative Commons licence, and indicate if changes were made.

The images or other third party material in this article are included in the article's Creative Commons licence, unless indicated otherwise in a credit line to the material. If material is not in-cluded in the article's Creative Commons licence and your intended use is not permitted by statutory regulation or exceeds the permitted use, you will need to obtain permission directly from the copyright holder.

To view a copy of this licence, visit http://creativecommons.org/licenses/by/4.0/.

\section{References}

[1] Taylor D O, Edwards L B, Boucek M M, Trulock E P, Keck B M, Hertz M I. The registry of the International Society for Heart and Lung Transplantation: Twenty-first official adult heart transplant report-2004. J Heart Lung Transplant 23(7): 796-803 (2004)

[2] Frazier O H, Rose E A, Oz M, Dembitsky W, McCarthy P,
Radovancevic B, Poirier V L, Dasse K A, HeartMate LVAS Investigators. Multicenter clinical evaluation of the HeartMate vented electric left ventricular assist system in patients awaiting heart transplantation. $J$ Thorac Cardiovasc Surg 122(6): 1186-1195 (2001)

[3] Joyce L D, Noon G P, Joyce D L, DeBakey M E. Mechanical circulatory support - a historical review. ASAIO J 50(6): x-xii (2004)

[4] Slaughter M S, Tsui S S, El-Banayosy A, Sun B C, Kormos R L, Mueller D K, Massey H T, Icenogle T B, Farrar D J, Hill J D, et al. Results of a multicenter clinical trial with the thoratec implantable ventricular assist device. $J$ Thorac Cardiovasc Surg 133(6): 1573-1580.e2 (2007)

[5] El-Banayosy A, Arusoglu L, Kizner L, Tenderich G, Minami K, Inoue K, Körfer R. Novacor left ventricular assist system versus HeartMate vented electric left ventricular assist system as a long-term mechanical circulatory support device in bridging patients: A prospective study. $J$ Thorac Cardiovasc Surg 119(3): 581-588 (2000)

[6] Farrar D J, Hill J D, Pennington D G, McBride L R, Holman W L, Kormos R L, Esmore D, Gray Jr L A, Seifert P E, Schoettle G P, et al. Preoperative and postoperative comparison of patients with univentricular and biventricular support with the Thoratec ventricular assist device as a bridge to cardiac transplantation. J Thorac Cardiovasc Surg 113(1): 202-209 (1997)

[7] Baughman K L, Jarcho J A. Bridge to life-cardiac mechanical support. N Engl J Med 357(9): 846-849 (2007)

[8] Rogers J G, Aaronson K D, Boyle A J, Russell S D, Milano C A, Pagani F D, Edwards B S, Park S, John R, Conte J V, et al. Continuous flow left ventricular assist device improves functional capacity and quality of life of advanced heart failure patients. J Am Coll Cardiol 55(17): 1826-1834 (2010)

[9] Eisen H J, Hankins S R. Continuous flow rotary left ventricular assist device. J Am Coll Cardiol 54(4): 322-324 (2009)

[10] Miller L W, Pagani F D, Russell S D, John R, Boyle A J, Aaronson K D, Conte J V, Naka Y, Mancini D, Delgado R M, et al. Use of a continuous-flow device in patients awaiting heart transplantation. N Engl J Med 357(9): 885-896 (2007)

[11] Pagani F D, Miller L W, Russell S D, Aaronson K D, John R, Boyle A J, Conte J V, Bogaev R C, MacGillivray T E, Naka $Y$, et al. Extended mechanical circulatory support with a continuous-flow rotary left ventricular assist device. $\mathrm{J} \mathrm{Am}$ Coll Cardiol 54(4): 312-321 (2009)

[12] Kirklin J K, Naftel D C, Kormos R L, Pagani F D, Myers S L, Stevenson L W, Acker M A, Goldstein D L, Silvestry S C, Milano C A, et al. Interagency registry for mechanically assisted circulatory support (INTERMACS) analysis of pump thrombosis in the HeartMate II left ventricular assist 
device. J Heart Lung Transplant 33(1): 12-22 (2014)

[13] Wu W T, Yang F, Wu J C, Aubry N, Massoudi M, Antaki J. High fidelity computational simulation of thrombus formation in Thoratec HeartMate II continuous flow ventricular assist device. Sci Rep 6: 38025 (2016)

[14] Tu J Y, Yeoh G H, Liu C Q. Computational Fluid Dynamics: A Practical Approach. 2nd ed. Oxford (Britain): ButterworthHeinemann, 2012.

[15] Zhang T T, Gao B, Zhou Z X, Chang Y. The movement and deposition of $\mathrm{PM}_{2.5}$ in the upper respiratory tract for the

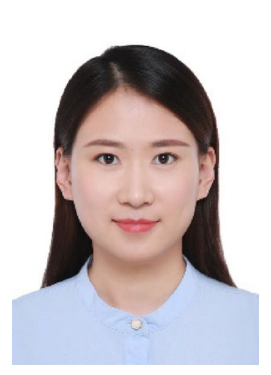

Xiaobing ZHENG. She received her bachelor degree in biological science from Hebei University, Hebei, China, in 2016. Now, she is a master student majoring in

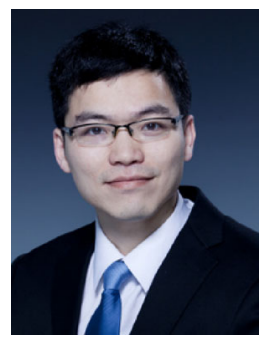

Yongjian LI. He received his Ph.D. degree in mechanical engineering from Tsinghua University, China, in 2009. He joined the Department of Mechanical Engineering at

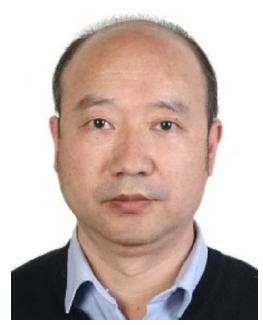

Jianye ZHOU. He received his master degree in biomedical engineering from Peking Union Medical College in 1995. He joined Fu Wai hospital, Chinese Academy of Medical Sciences from 1998 as a patients with heart failure: An elementary CFD study. BioMed Eng On Line 15 (S2): 138 (2016)

[16] Chiang H L, Hsu C H, Lin J R. Lubrication performance of finite journal bearings considering effects of couple stresses and surface roughness. Tribol Int 37(4): 297-307 (2004)

[17] Chang L. Deterministic modeling and numerical simulation of lubrication between rough surfaces - a review of recent developments. Wear 184(2): 155-160 (1995)

[18] Freund J B. Numerical simulation of flowing blood cells. Annu Rev Fluid Mech 46: 67-95 (2014)

biochemistry and molecular biology at State Key Laboratory of Cardiovascular Disease in Peking Union Medical College, Beijing, China. Her research areas are biomaterials and artificial organs.

Tsinghua University from 2011. His current position is an assistant professor. His research areas cover the tribology in seals and bearings, transport phenomena and interfacial behaviors in microfluidics and bio-chips.

scientific researcher. His current position is a professor and the deputy chief of the Laboratory of Cardiovascular Regenerative Medicine. His research area covers development of implantable devices, anti-calcification strategies, and cardiovascular tissue engineering. 